\title{
Formation of Extra Vacancies in Nucleating $\gamma$ Phase during $\delta$ - $\gamma$ Massive-like Phase Transformation in Carbon Steel
}

\author{
Masato YOSHIYA* , Katsuya SHIRAI* and Hideyuki YASUDA**
}

(Received 17 April 2021, Accepted 21 April 2021)

\begin{abstract}
Possible formation of extra amount of Fe vacancies during the massive-like phase transformation of carbon steel has been examined by combinations of analytical modeling, atomistic simulations and phase-field modeling. Formation of a vacancy in a perfect crystal does not decrease its volume by as much as the volume of an atom, effectively increasing lattice constant per atom. Consequently, transformation strain due to solid-state transformation from BCC-structured $\delta$ phase to FCC-structured $\gamma$ phase leads to formation of extra amount of $\mathrm{Fe}$ vacancies more than in thermal equilibrium. This is to relieve transformation strain due to nucleation and growth of $\gamma$ phase, which would promotes the massive-like transformation. The extra vacancies may also promote diffusion of substitutional alloying elements across $\delta / \gamma$ interface or partitioning of the alloying elements during the massive-like transformation, at least in the nucleating $\gamma$ phase and in the vicinity of $\delta / \gamma$ interface until transformation strain is relieved.
\end{abstract}

Key Words: Interface Energy, Vacancies, Solidification, Soild-state Transformation, Carbon Steel

\section{Introduction}

Microstructures of materials are critically important as they determine overall materials properties in macroscopic dimension, even though they originated from atomistic level or electronic structures. Phase transformation initiates microstructures evolution and thus its careful and precise control is crucial for tailoring materials for a wide spectrum of practical applications. It is even more so for additive manufacturing as it accompanies rapid melting and solidification led by precisely controlled energy probe, such as electron or laser beam. For the careful and precise control, in-depth understanding as to what is happening during phase transformations is necessary ${ }^{1-5)}$. In this light, computeraided simulations, theoretical calculations, and numerical and mathematical analyses have been intensively and extensively done for many decades.

Carbon steel is one of the most extensively and intensively studied materials. Yet, recent in situ observation of its phase transformation using synchrotron radiation with high coherency and brightness has revealed that a solid-state phase transformation from BCC-structured less dense $\delta$ phase to FCC-structured dense $\gamma$ phase occurs following solidification from the liquid phase $^{6-11)}$. At the temperature range, peritectic reaction is expected according to binary phase diagram ${ }^{12}$. This phase transformation can be easily distinguished from the peritectic reaction in many aspects. First of all, it does not involve liquid phase, which can be confirmed by shrinkage over wide space reflecting the difference in volumes or densities between the two phases. In an analogy to the massive transformation which is defined as compositional invariance $^{13)}$, this solid state transformation is then referred to as massive-like transformation ${ }^{9-11)}$.

Careful and systematic in situ observations of the massivelike phase transformation revealed many differences from the peritectic reaction ${ }^{11}$. For example, (i) it requires higher undercooling relative to peritectic temperature and (ii) the phase transformation completes at significantly faster rates than the peritectic reaction. In addition, it is found that (iii) interface morphology between $\delta$ phase and $\gamma$ phase is highly dissimilar to the ones of diffusion-controlled phase transformations, that (iv) curves on the phase diagram do not separate the phenomena, and that (v) it is observed also in stainless steels, by careful experiments. Even though those experimental approaches revealed possible mechanisms behind the massive-like phase transformation, it inevitably has limitations to further uncover the mechanisms even by cutting-edge experiments.

Thus, in parallel, we have employed computational approaches as well. Starting with a systematic atomistic simulations using semi-classical interatomic force field, i.e., embedded atom method (EAM) which accounts for electronic structures in a parameterized form, interface energies not only of homogeneous phase boundaries, i.e., grain boundaries, but also of heterogeneous $\delta / \gamma$ interfaces have been quantified as a function of misorientation across the interface. To quantify representative values of each interface energy for further analyses, phase

* Division of Materials and Manufacturing Science, Graduate School of Engineering, Osaka University (2-1 Yamadaoka, Suita, Osaka 565-0871, Japan) * * Department of Materials Science and Engineering, Kyoto University (Yoshida-honmachi, Sakyo, Kyoto 606-8501, Japan) 
field modeling $(\mathrm{PFM})^{14)}$ for grain growth was used. With the representative values for each interface, a simple numerical analysis has performed, which explains (i) the necessity of the high undercooling to initiate the massive transformation and the reason behind (ii) the faster rate of the transformation in terms of concurrent nucleations. The concurrent nucleations is enabled by reduced undercooling needed for secondary and later nucleations following the initial nucleations ${ }^{15)}$. This explains (iii) the peculiar interface morphology observed by the in situ observation with high temporal resolution ${ }^{10}$. Influence of transformation strain from $\delta$ phase and $\gamma$ phase is also examined ${ }^{16)}$. These findings obtained by the simple numerical analysis have been demonstrated by PFM simulations with thermal fluctuation to enable nucleation in the course of simulations ${ }^{17}$. It also explains a possible reason why the nucleation of $\gamma$ phase dominates the massive-like phase transformation, instead of growth of the nucleated $\gamma$ phase. Furthermore, it is demonstrated that the growth of the $\gamma$ phase "region" is suppressed and it is nucleationcontrolled phase transformation ${ }^{18)}$.

These computational studies are limited to alloying element free steels, being unable to address further issues, such as (iv) and (v). For alloying elements to be partitioned during the massive transformation or afterwards, their diffusion is necessary which requires $\mathrm{Fe}$ vacancies. However, it remain challenging to directly detect temporal creation or annihilation of vacacies away from thermodynamic equilibrium even by the cutting-edge experiments. Thus, in this study, we attempted to uncover the presence and roles of vacancies during the massive-like phase transformation or afterwards, as vacancies are not accounted for in preceding studies, even though it is easily expected that vacancies are easily created, migrated, and annihilated at the elevated temperature.

\section{Methods}

In this study, conventional phase-field modeling (PFM) according to Kim-Kim-Suzuki (KKS) scheme ${ }^{19)}$ for descriptions of interfaces and to Steinbach's multi-phase field modeling $(\mathrm{MPFM})^{20)}$ to deal with multiple phase-field variables is employed. The former determines parameters necessary for the PFM solely from interface energy and a prescribed interface thickness. The latter requires the sum of phase field variables at a local grid point to be unity.

The PFM is based on non-equilibrium thermodynamics ${ }^{21)}$ where it is assumed that local equilibrium holds within a local volume, or all the theories of equilibrium thermodynamics hold in the local volume, in other words, and that transport phenomena including motion of interfaces occur to minimize overall free energy of the target system. A phase-field variable, $\phi_{i}(\mathbf{r})$, is defined for each phase and it denotes a probability of existence of the phase at a position, $\mathbf{r}$. Its time evolution is calculated in PFM as

$$
\frac{\partial \phi_{i}}{\partial t}=-M \frac{\delta f(\mathbf{r}, \mathrm{T})}{\delta \phi_{i}}
$$

where $f(\mathbf{r}, T)$ is local free energy of a small volume at a position $\mathbf{r}, t$ is time, $M$ is mobility of interface and $\delta$ on the right hand side of the equation denotes variation instead of partial differentiation. Helmholtz free energy instead of Gibbs free energy was used for a constant volume condition or a fixed mesh grids. In principle, any energy factor can be included in the local free energy to account for any physical phenomena of interest. In this study, local free energy, $f$, is defined as

$$
f=f^{\mathrm{vol}}+f^{\text {strain }}+f^{\text {interface }}+f^{\mathrm{vac}}
$$

Volumetric free energy, $f^{\mathrm{vol}}$, of $\delta$ and $\gamma$ phases were calculated from the approximated equation:

$$
f^{\mathrm{vol}}=-\left(\mathrm{T}_{\mathrm{P}}-T\right) \Delta S_{\gamma \rightarrow \delta}=-\left(\mathrm{T}_{\mathrm{P}}-T\right)\left(S_{\delta}-S_{\gamma}\right)
$$

where $\mathrm{T}_{\mathrm{P}}$ is the peritectic temperature and $S_{\delta}$ and $S_{\gamma}$ are entropy which are taken from a thermodynamic database ${ }^{22)}$ and used after converting its unit from $\mathrm{kJ} / \mathrm{mol}$ to $\mathrm{kJ} / \mathrm{m}^{3}$ using equilibrium lattice constants shown in Table 1. Elastic strain energy, $f^{\text {strain }}$, is calculated using the Lame's constants for the cubic phases calculated by atomistic simulations in the preceding study. Due to its long range nature of the elastic interaction, calculations of elastic strain energy was done based on micromechanics ${ }^{23)}$ in Fourier space. Transformation strain between the two phases were calculated from the equilibrium lattice constants in Table 1. Interface energies, $f^{\text {interface }}$, between the same phase (grain boundaries) or the two different phases were also calculated by atomistic simulations in the previous study ${ }^{24)}$, which are tabulated in Table 2. Formation free energy of Fe vacancies, $f^{\text {vac }}$, which was also calculated by atomistic simulations in this study was also added to overall local free energy.

In this study, vacancies are described with a non-conservative

Table 1 Phase specific parameters calculated by atomistic simulations.

\begin{tabular}{|l|c|c|c|}
\hline & $\delta$ phase & $\gamma$ phase & Ref. \\
\hline Lattice Constant, $a[\AA]$ & 2.9322 & 3.6468 & {$[32]$} \\
\hline Entropy, S $[\mathrm{J} / \mathrm{K} \cdot \mathrm{mol}]$ & 88.721 & 88.218 & {$[22]$} \\
\hline Lame's constant, $\lambda[\mathrm{GPa}]$ & 141 & 131 & {$[17]$} \\
\hline Lame's constant, $\mu[\mathrm{GPa}]$ & 42.9 & 15.9 & {$[17]$} \\
\hline
\end{tabular}

Table 2 Other parameters used in PFM.

\begin{tabular}{|l|c|c|}
\hline & & Ref. \\
\hline Peritectic Temperature $[\mathrm{K}]$ & 1766 & {$[12]$} \\
\hline Effective Interface Energy, $\sigma_{\gamma / \gamma}\left[\mathrm{J} / \mathrm{m}^{2}\right]$ & 0.44 & {$[24]$} \\
\hline Effective Interface Energy, $\sigma_{\delta / \delta}\left[\mathrm{J} / \mathrm{m}^{2}\right]$ & 0.37 & {$[24]$} \\
\hline Effective Interface Energy, $\sigma_{\delta / \gamma}\left[\mathrm{J} / \mathrm{m}^{2}\right]$ & 0.56 & {$[24]$} \\
\hline Mobility of Interface, $M\left[\mathrm{~m}^{4} / \mathrm{J} \cdot \mathrm{s}\right]$ & $4.0 \times 10^{-14}$ & \\
\hline Spatial Mesh Grid Intervals $[\AA]$ & 10 & \\
\hline Prescribed Interface Thickness $[$ grid] & 4 & \\
\hline
\end{tabular}


variable. In other words, a separate phase-field variable is defined for vacancies to discuss spatial distribution of vacancies and its time evolution accompanied by the $\delta$ to $\gamma$ phase transformation. By doing so, this study aims at discussing creation and annihilation of vacancies in the course of the $\delta-\gamma$ phase transformation. Although diffusion of vacancies can be coupled with the governing equation of PFM through local free energies, the diffusion of vacancies were neglected in this study.

In the PFM simulations, interplay or competition among aforementioned energy factors is important. Thus, atomistic simulations with static energy minimization technique were done using the same interatomic interaction described by embedded atom method (EAM) which accounts for charge density parametrized in prior to calculations. Parameters reported by Dai et al. ${ }^{25)}$ were used in this study and calculations were done using LAMMPS $\operatorname{code}^{26)}$. The Lame's constants were calculated by applying various external stresses to the supercells. In this study, 500 atom-containing supercells were used to calculate vacancy formation enthalpy and configurational entropy was calculated based on the supercell. The obtained enthalpy is in good agreement with the value separately calculated in this study by $a b$ initio calculations with generalized gradient approximation (GGA) exchange-correlation functional ${ }^{27,28)}$ using VASP $\operatorname{code}^{29-31}$. Spin polarization was neglected as it is plausible to assume that it affects little at elevated temperatures. Interface energies, whether between the same phases or different phases, were calculated as a function of misorientation angle across the interface, and effective interface energies for $\delta / \gamma$ interface, $\delta / \delta$ grain boundaries, $\gamma / \gamma$ grain boundaries were evaluated using PFM simulations for grain growth by assigning different phase-field variables even for the same thermodynamic phase having different crystallographic orientations $^{24)}$.

\section{Results and Discussion}

According to a classical nucleation theory ${ }^{13)}$, critical radius, $\mathrm{r}^{*}$, is given (in the case of two dimensions) by

$$
\mathrm{r}^{*}=\frac{\sigma_{\delta / \gamma}}{\left(\mathrm{T}_{\mathrm{P}}-T\right)\left(S_{\delta}-S_{\gamma}\right)}
$$

Thus, at $1200 \mathrm{~K}$, the critical radius is analytically (mathematically) calculated to be $14.36 \mathrm{~nm}$. In the PFM, no such theory as this but fundamental thermodynamics is explicitly included in the governing equation. To demonstrate whether the critical radius is reproduced or not, PFM simulations for $\gamma$ phase nucleus/embryo embedded in $\delta$ phase with initial radius of nucleus being around the critical radius were performed. As shown in Fig. 1, when the initial radius, $r^{\text {init }}$, is smaller than the critical radius, the nucleaus/ embryo gradually decreased its size and eventually disappeared. On the other hand, when the initial radius is greater than the critical radius, nucleus grew with time. Although elastic strain energy is not taken into account in this preliminary calculations, it has been confirmed that inclusion elastic strain energy does not show discrepancy with analytical solutions when analytical solutions are available.

Calculations of formation internal energies of a Fe vacancy in BCC-structured $\delta$ phase and in FCC-structured $\gamma$ phase were done both by semi-classical atomistic simulations of static energy minimization technique with EAM and by $a b$ initio calculations with one vacancy introduced to $5 \times 5 \times 5$ supercells containing 500 atoms when there is no vacancy in the case of $\gamma$ phase (Fig. 2). The calculations were done also for smaller supercell containing 108 atoms. As discussion in this study is for high temperature, spatial distribution of initial magnetic moment were not set in prior to $a b$ initio calculations. All the calculations were done under constant pressure condition, i.e., supercell volumes were also relaxed together with all the atomic positions upon structure optimizations.

Values of vacancy formation enthalpy, which are equal to formation internal energy in this study as contribution from PV term is small enough to neglect, for $\delta$ phase and $\gamma$ phase are

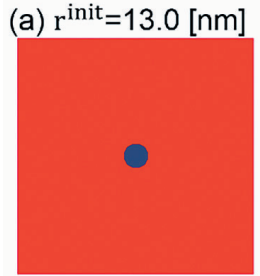

0 [step]

(b) $\mathrm{r}^{\text {init }}=15.0[\mathrm{~nm}]$

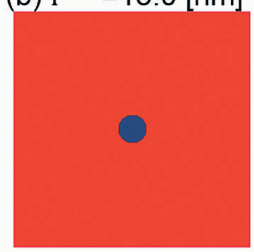

0 [step]

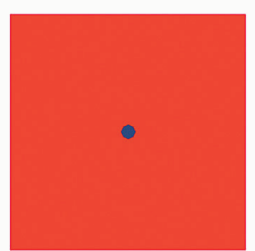

1500 [step]

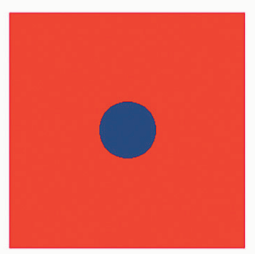

5000 [step]

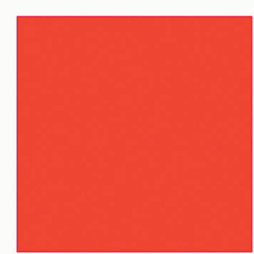

1800 [step]

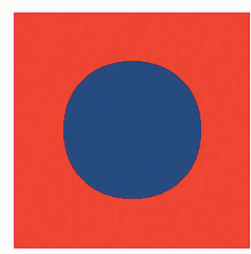

10000 [step]
Fig. 1 Time evolution of $\gamma$ phase nucleus/embryo embedded in $\delta$ phase matrix phase in the absence of elastic strain energy.

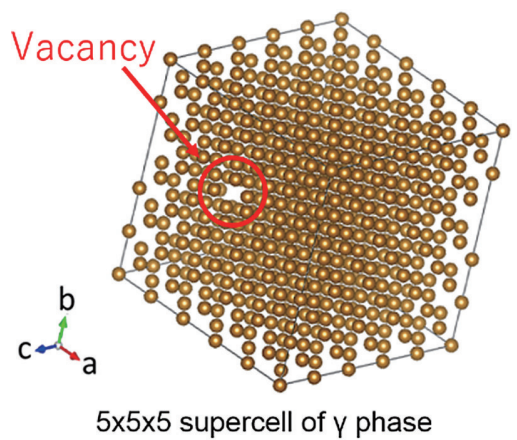

Fig. 2 A $5 \times 5 \times 5$ supercell of $\gamma$ phase with a vacancy, which contains 499 atoms. 
1.84 and $1.82 \mathrm{eV}$, respectively, for the vacancy concentration of one out of 500. The value for $\gamma$ phase is almost unchanged even when supercell size is reduced to 108 atoms. In both cases, these values are in good agreements with the values obtained by $a b$ initio calculations. It also has a satisfactory agreement with an experimental value measured, $1.7 \mathrm{eV}$ at $1100 \mathrm{~K}^{33}$. It should be noted that vacancy concentration discussed in this study is far below the concentration in the supercell. In that case, separation between vacancies are farther and thus it is plausible to assume that vacancy formation internal energy is constant for the realistic concentration discussed below. Since there is no significant difference in the formation internal energy between the two phases, hereafter vacancy formation internal energy of $\gamma$ phase are used also for $\delta$ phase for simplicity and clarity. This formation internal energy does not contain PV term and entropy term to be a free energy. Thus, configurational entropy calculated with the 500 -atom supercell and vibrational entropy taken from literature ${ }^{34)}$ are used to form vacancy formation free energy as a function of temperature. Since contributions of values of PV terms to free energy is negligibly small in this study, the PV term is set to zero in this study.

With the vacancy formation free energy per vacancy, free energy difference upon formation of vacancies at $1400 \mathrm{~K}$ are analytically calculated as a function of vacancy concentration. As shown in Fig. 3, the free energy difference has minima at far lower than that in the supercell used in the atomistic simulation. The minimum of the red curve corresponds to thermal equilibrium concentration of vacancies. On the other hand, the minimum of the blue curve corresponds to the equilibrium vacancy concentration under transformation strain from $\delta$ phase to $\gamma$ phase.

Since $\gamma$ phase is close-pack structure while $\delta$ phase is sparse BCC structure, nucleated $\gamma$ phase attempts to reduce its volume upon solid state phase transformation. However, formation of void between the two phases energetically costs by the surface energies of both phases, inhibiting creation of voids. Upon formation of a vacancy, volume change of a phase is not as much as volume per atom. Thus, formation of vacancies efffectively increases volume per atom of the dense FCC-structured $\gamma$ phase, or it increases lattice constant per atom. This will reduce the energy penalty by the transformation strain. Thus, under the transformation strain, equilibrium vacancy concentration is increased more than the thermal equilibrium value.

The equilibrium vacancy concentration with or without the transformation strain are for uniform phase without considering spatial variation of vacancy concentration in the system. However, it is expected that when the two phases coexist and the nucleated $\gamma$ phase grow, it is possible that the system deviates from thermodynamic equilibrium with elastic strain therein. To demonstrate that the extra vacancy is necessary for the nucleated

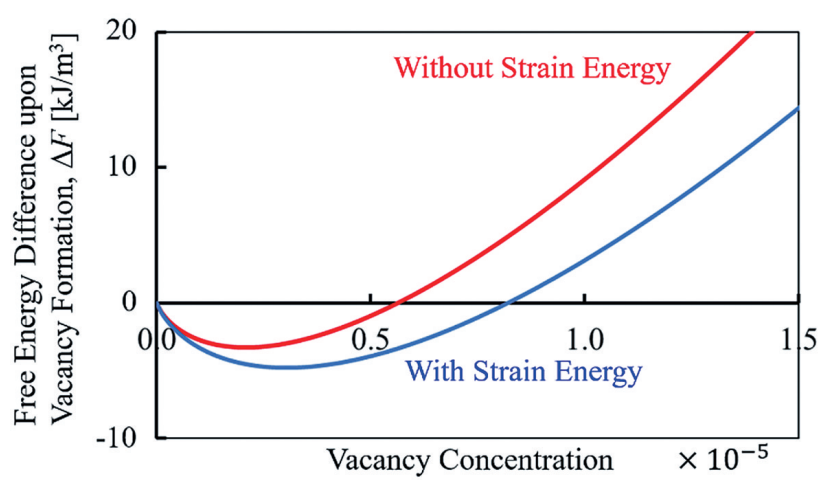

Fig. 3 Changes in free energies upon vacancy formations in $\gamma$ phase as a function of vacancy concentration. Red curves are obtained by neglecting elastic strain energy, and thus transformation strain from BCC to FCC. Blue curves are obtained by taking into account the elastic strain energy, corresponding to the formation under transformation strain from BCC to FCC.

$\gamma$ phase to be stabilized, PFM simulations with prescribed nucleus greater than its critical radius were done with $256 \times 256$ grid two dimensional mesh. As critical radius increases to $36.1 \mathrm{~nm}$ under the transformation strain, initial nucleus of $60 \mathrm{~nm}$ in radius was placed at the center of the simulation model.

For the PFM simulations, two technical problems need to be bypassed. The first one is too small equilibrium vacancy concentration (Fig. 3) compared with numerical noises, and the second one is the necessity to account for interface energy between vacancies and neighboring real phases when vacancies are treated as if it is a phase using a phase-field variable. The latter problem results in artificially uniform distribution of vacancies to minimize gradient energy. However, the latter problems can be bypassed by partitioning the vacancy formation free energy, $f^{\text {vac }}$, into two terms using a partitioning parameter, $\alpha$ :

$$
f^{\mathrm{vac}}=V \cdot\left(H^{\mathrm{vac}} \cdot \alpha-T \Delta S_{\delta \rightarrow \gamma}\right)+A \cdot H^{\mathrm{vac}} \cdot(1-\alpha) \mathrm{a}_{\gamma}
$$

where $\mathrm{a}_{\gamma}$ is the lattice constant of $\gamma$ phase shown in Table 1, volume, $V$, and surface area, $A$, only to adjust unit in the equation: within the frame work of the PFM, $V$ or $A$ do not have to be explicitly calculated and they are given only by spatial distribution of phase-field variables, which is a virtue of the PFM. The entropy term is give only to the first term on the right hand side of the equation. The first term on the right hand side of the equation corresponds to volumetric free energy of vacancies and the second term corresponds to interface energy between vacancies, or vacuum, and $\delta$ or $\gamma$ phase. Although it is possible to uniquely determine the partitioning parameter, $\alpha$, by adjusting $\alpha$ such that the interface energy would be surface energy between $\delta$ or $\gamma$ phase and vacuum. However, the first problem persists in that case. Thus, by sacrificing to some extent the accuracy of surface energy which becomes important when vacancies agglomerate to form a void although it has proven too energetically expensive ${ }^{16)}$, $\alpha$ was set to 0.12 according to preliminary calculations. This $\alpha$ 


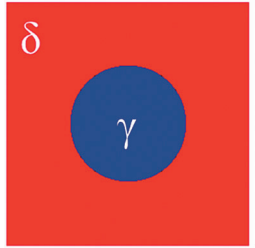

1000 [step]

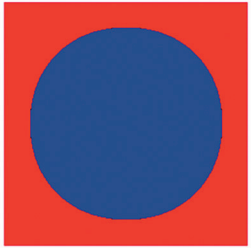

20000 [step]

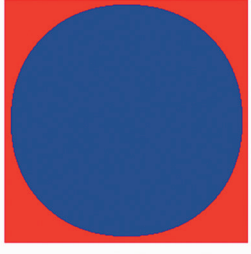

25000 [step]
Fig. 4 Growth of $\gamma$ phase nucleus in $2.5 \times 2.5 \mu \mathrm{m}^{2} \delta$ matrix phase.

value yields thermal equilibrium vacancy concentration of 0.004 for both $\delta$ and $\gamma$ phases due to the same formation free energy used, which is far greater than the values analytically estimated (Fig. 3) because of the partitioning parameter $\alpha$. Different $\alpha$ value results in different vacancy concentrations and thus attention should be paid only to the relative change in vacancy concentrations.

As the initial radius of prescribed $\gamma$ phase nucleus is greater than its critical radius at $1400 \mathrm{~K}$ which corresponds to undercooling of $366 \mathrm{~K}$, the $\gamma$ phase kept growing with time (Fig. 4). As seen from Fig. 5, vacancy concentration within the $\gamma$ phase nucleus is 0.0063 at 1000 step, significantly higher than the equilibrium concentration for the $\alpha$ value used. This indicates that extra amount of vacancies are created within the nucleated $\gamma$ phase to minimize the energy penalty by the transformation strain from BCC to FCC structures. Its vacancy concentration decreases as the $\gamma$ phase grows, reaching 0.0049 at 25000 step. On the other hand, the lower amount of vacancies than thermal equilibrium were created or vacancy concentration was decreased from the initial concentration in the $\delta$ phase. This can be attributed to the strain imposed by the nucleated $\gamma$ phase. With the dominance of $\gamma$ phase upon its growth, vacancy concentration in the $\delta$ phase was further decreased. Although segregation of vacancies at $\delta / \gamma$ interface were observed in the PFM simulation in this study, further studies are needed to quantitatively address the possible segregation as it may be due to the shortcomings of the PFM scheme used in this study, as pointed out by Ohno and Matsuura ${ }^{35)}$.

This trend of extra vacancies is presumably overestimated in this PFM simulations due to the partitioning parameter and also to limited size of the simulation box. However, it is expected that this trends remain the same even if greater size of simulation box is used, and also in experiments.

The formation of extra vacancies due to $\gamma$ phase nucleation and growth indicates that diffusion not only of carbon at interstitial sites but also of substitutional elements are promoted by the extra amount of vacancies, at least in nucleated $\gamma$ phase and in the vicinity of $\delta / \gamma$ interfaces until transformation strain is relieved. Although it is beyond the scope of the present study, it is of great interest to directly examine diffusions of substitutional elements across the $\delta / \gamma$ interfaces or non-equilibrium partitioning of the

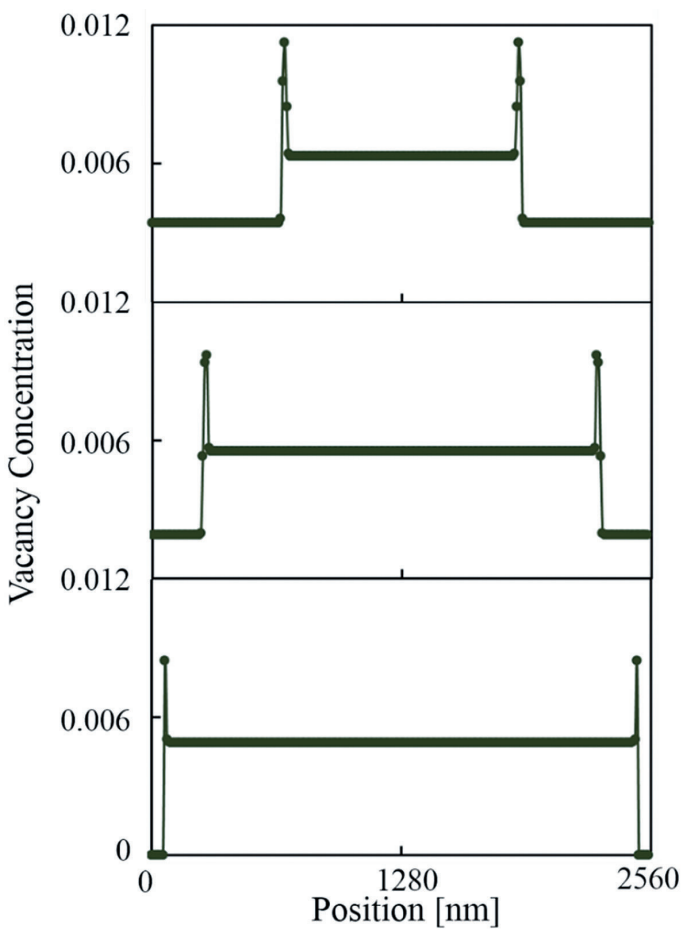

Fig. 5 Time evolution of line profile of vacancy concentration across the center of $\gamma$ phase nucleus.

substitutional elements in the massive-like transformation.

\section{Conclusions}

In this study, formation of $\mathrm{Fe}$ vacancies during the massive-like phase transformation in carbon steel is examined by a combination of analytical modeling, atomistic simulations and phase-field modeling. Atomistic simulations revealed that formation of a vacancy does not decrease the volume of a phase by as much as volume per atom, which effectively increase the lattice constant per atom. Transformation strain from BCC-structured $\delta$ phase to dense FCC structured $\gamma$ phase in the massive-like transformation thus leads to formation of extra amount of Fe vacancies, more than thermal equilibrium, to relieve transformation strain thereby minimizing the strain energy. The extra amount of vacancies may promote diffusion of substitutional alloying elements, at least in nucleated $\gamma$ phase and in the vicinity of $\delta / \gamma$ interfaces until transformation strain is relieved.

\section{Acknowledgment}

This study is supported by Grant-in-Aid for Scientific Research S (Grant Number: JP17H06155).

\section{References}

1) R.H. Mathiesen, L. Arnberg, F. Mo, T. Weitkamp and A. Snigirev: "Time Resolved X-Ray Imaging of Dendritic Growth in Binary Alloys", Phys. Rev. Lett., 83 (1999), 5062-5605.

2) H. Yasuda, I. Ohnaka, K. Kawasaki, A. Sugiyama, T. Ohmichi, J. 
Iwane and K. Umetani: "Direct observation of stray crystal formation in unidirectional solidification of Sn-Bi alloy by X-ray imaging", J. Cryst. Growth, 262 (2004), 645.

3) N. Mangelinck-Noel, H. Nguyen-Thi, G. Reinhart, T. Schenk, V. Cristiglio, M.D. Dupouy, J. Gastaldi, B. Billia and J. Hartwig: "In situ analysis of equiaxed growth of aluminium-nickel alloys by $\mathrm{x}$-ray radiography at ESRF", J. Phys. D, 38 (2005), A28-A32.

4) B. Li, H. D. Brody, D. R. Black, H. E. Burdette and C. Rau: "Real time observation of dendritic solidification in alloys by synchrotron microradiography", J. Phys. D: Appl. Phys., 39 (2006), 4450-4456.

5) T.M. Wang, J.J. Xu, T.Q. Xiao, H.L. Xie, J. Li, T.J. Li and Z.Q. Cao: "Evolution of dendrite morphology of a binary alloy under an applied electric current: An in situ observation", Phys. Rev. E, 81 (2010), 042601-1-4.

6) H. Yasuda, Y. Yamamoto, N. Nakatsuka, T. Nagira, M. Yoshiya, A. Sugiyama, I. Ohnaka, K. Umetani and K. Uesugi: "In situ observation of solidification phenomena in Al-Cu and Fe-Si-Al alloys", Int. J. Cast Met. Res., 22 (2008), 15-21.

7) H. Yasuda, T. Nagira, M. Yoshiya, N. Nakatsuka, A. Sugiyama, K. Uesugi and K. Umetani: "Development of X-ray Imaging for Observing Solidification of Carbon Steels", ISIJ Int., 51 (2011), 402-408.

8) T. Nagira, C.M. Gourlay, A. Sugiyama, M. Uesugi, Y. Kanazawa, M. Yoshiya, K. Uesugi, K. Umetani and H. Yasuda: "Direct observation of deformation in semi-solid carbon steel", Scr. Mater., 64 (2011), 1129-1132.

9) H. Yasuda, T. Nagira, M. Yoshiya, A. Sugiyama, N. Nakatsuka, M. Kiire, M. Uesugi, K. Uesugi, K. Umetani and K. Kajiwara: "Massive transformation from $\delta$ phase to $\gamma$ phase in Fe.C alloys and strain induced in solidifying shell", IOP Conf. Ser.: Mater. Sci. Eng., 33 (2012), 012036-1-8.

10) T. Nishimura, K. Morishita, T. Nagira, M. Yoshiya and H. Yasuda: "Kinetics of the $\delta / \gamma$ interface in the massive-like transformation in Fe-0.3C-0.6Mn-0.3Si alloys", IOP Conf. Ser. Mater. Sci. Eng., 84 (2015), 012062.

11) T. Nishimura, R. Matsubayashi, K. Morishita, M. Yoshiya, T. Nagira and H. Yasuda: "Selection of the Massive-like $\delta-\gamma$ Transformation due to Nucleation of Metastable $\delta$ Phase in Fe-18 Mass\%Cr-Ni Alloys with Ni Contents of 8, 11, 14 and 20 Mass\%", ISIJ Int., 59 [3] (2019), 459-465.

12) T.B. Massalski, J.L. Murray, L.H. Bennett and H. Baker (Eds.), "Binary Alloy Phase Diagrams", Americal Society of Metals, (1986), 563.

13) J.A. Dantzig and M. Rappz: "Solidification", EPEL Press, (2009), 249-253.

14) R. Kobayashi, "Modeling and numerical simulations of dendritic crystal growth", Physica D, 63 (1993), 410-423.

15) M. Yoshiya, M. Watanabe, K. Nakajima, N. Ueshima, K. Hashimoto, T. Nagira and H. Yasuda: "Concurrent $\delta$-Phase Nucleation as a Possible Mechanism of $\delta-\gamma$ Massive-like Phase Transformation in Carbon Steel: Numerical Analysis based on Effective Interface Energy", Mater. Trans., 56 (2015), 1467-1474.

16) M. Yoshiya, M. Sato, M. Watanabe, K. Nakajima, T. Yokoi, N. Ueshima, T. Nagira and H. Yasuda: "Impacts of Interface Energies and Transformation Strain from BCC to FCC on Massive-like $\delta$ - $\gamma$ Transformation in Steel", IOP Conf. Ser.: Mater. Sci. Eng., 84 (2015),
012049-1-8.

17) H. Fujiwara: "Analyses of constituent energy factors upon $\delta-\gamma$ massive-like transformation by phase field modeling with nucleation", Master Thesis, Osaka University, Japan, (2017), 28-96.

18) K. Kurotsu: "Influence of multi-level inhomogeneity on $\delta-\gamma$ massive-like transformation of carbon steel", Master Thesis, Osaka University, Japan, (2019), 20-97.

19) S.G. Kim, W.T. Kim and T. Suzuki: "Phase-field model for binary alloys", Phys. Rev. E, 60 (1999), 7186-7197.

20) I. Steinbach and M. Apel: "Multi phase field model for solid state transformation with elastic strain", Physica D, 217 (2006), 153-160.

21) S.R. de Groot and P. Mazur: "Non-equilibrium thermodynamics", Dover Publications, (1984), 11-82.

22) M.W. Chase, Jr., C.V. Mock, J.F. Masi, F.E and Spencer, Jr. (Eds.): "NIST-JANAF Thermochemical Tables", Forth Edition, The American Chemical Society and The Americn Institute of Physics for The National Institute of Standards and Technology, (1998), 1221-1226, and references therein.

23) J. Qu and M. Cherkaoui: "Fundamentals of micromechanics of solids", John Wiley \& Sons, (2006), 11-195.

24) M. Yoshiya, K. Nakajima, M. Watanabe, N. Ueshima, T. Nagira and H. Yasuda: "Interface Energies of Hetero- and Homo-phase Boundaries and Their Impact on $\delta-\gamma$ Massive-like Phase Transformation in Carbon Steel", Mater. Trans., 56 (2015), 1461-1466.

25) X.D. Dai, Y. Kong, J.H. Li and B.X. Liu: "Extended Finnis-Sinclair potential for bcc and fcc metals and alloys", J. Phys.: Condens. Matter, 18 (2006), 4527-4542.

26) S. Plimton: "Fast parallel algorithms for short-range molecular dynamics", J. Comput. Phys., 117 (1995), 1-19.

27) J.P. Perdew, K. Burke and M. Ernzerhof: "Generalized gradient approximation made simple", Phys. Rev. Lett., 77 (1996), 3865 3868.

28) J.P. Perdew, K. Burke and M. Ernzerhof: "Errata: Generalized gradient approximation made simple [Phys. Rev. Lett. 77, 3865 (1996)]", Phys. Rev. Lett., 78 (1997), 1396.

29) G. Kresse and J. Hafner: "Ab inito molecular dynamics for liquid metals", Phys. Rev. B, 47 (1993), 558-561.

30) G. Kresse and J. Furthmüller: "Efficient iterative schemes for ab initio total-energy calculations using a plane-wave basis set", Phys. Rev. B, 54 (1996), 11169-11186.

31) P.E. Blöchl, "Projector augmented-wave method", Phys. Rev. B, 50 (1994), 17953-17979.

32) Nihon Kinzoku Gakkai: "Kinzoku deta bukku", Maruzen, (2004).

33) S. Kim and W. Buyers: "Vacancy formation energy in iron by positron annihilation", J. Phys. F: Met. Phys., 8 (1987), 67-70.

34) J.J. Burton: "Vacancy-formation entropy in Cubic Metals", Phys. Rev. B, 5 (1972), 2948-2957.

35) M. Ohno and K. Matsuura: "Quantitative phase-field modeling for dilute alloy solidification involving diffusion in the solid", Phys. Rev. E, 79 (2009), 031603-1-15.

\section{Mail Address}

Masato YOSHIYA yoshiya@mat.eng.osaka-u.ac.jp 